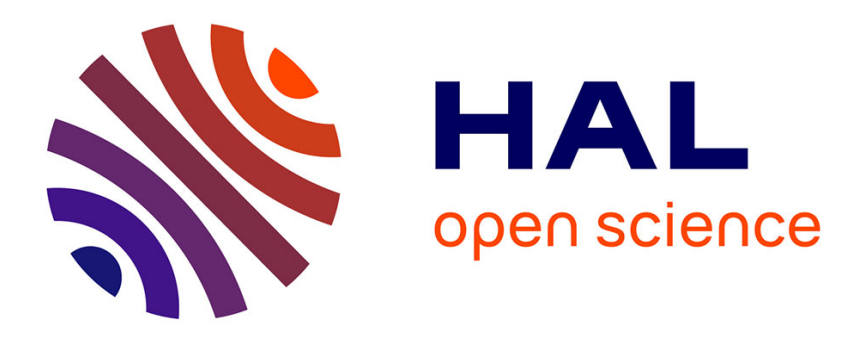

\title{
Finite-sample distribution of regression quantiles
}

Jana Jurečková

\section{- To cite this version:}

Jana Jurečková. Finite-sample distribution of regression quantiles. Statistics and Probability Letters, 2010, 80 (23-24), pp.1940. 10.1016/j.spl.2010.08.023 . hal-00691777

\section{HAL Id: hal-00691777 \\ https://hal.science/hal-00691777}

Submitted on 27 Apr 2012

HAL is a multi-disciplinary open access archive for the deposit and dissemination of scientific research documents, whether they are published or not. The documents may come from teaching and research institutions in France or abroad, or from public or private research centers.
L'archive ouverte pluridisciplinaire HAL, est destinée au dépôt et à la diffusion de documents scientifiques de niveau recherche, publiés ou non, émanant des établissements d'enseignement et de recherche français ou étrangers, des laboratoires publics ou privés. 


\section{Accepted Manuscript}

Finite-sample distribution of regression quantiles

Jana Jurečková

PII:

S0167-7152(10)00246-4

DOI:

10.1016/j.spl.2010.08.023

Reference: $\quad$ STAPRO 5782

To appear in: Statistics and Probability Letters

Received date: 8 June 2010

Revised date: 25 August 2010

Accepted date: 26 August 2010

Please cite this article as: Jurečková, J., Finite-sample distribution of regression quantiles.

Statistics and Probability Letters (2010), doi:10.1016/j.spl.2010.08.023

This is a PDF file of an unedited manuscript that has been accepted for publication. As a service to our customers we are providing this early version of the manuscript. The manuscript will undergo copyediting, typesetting, and review of the resulting proof before it is published in its final form. Please note that during the production process errors may be discovered which could affect the content, and all legal disclaimers that apply to the journal pertain. 


\title{
Finite-sample distribution of regression quantiles
}

\author{
Jana Jurečková*,1 \\ Department of Probability and Statistics, MFF UK, Charles University, \\ Sokolovská 83, CZ-186 75 Prague 8, Czech Republic
}

\begin{abstract}
The finite sample distributions of the regression quantile and of the extreme regression quantile are derived for a broad class of distributions of the model errors, even for the non-i.i.d case. The distributions are analogous to the corresponding distributions in the location model; this again confirms that the regression quantile is a straightforward extension of the sample quantile. As an application, the tail behavior of the regression quantile is studied.
\end{abstract}

Key words: order statistic, regression quantile, extreme regression quantile, regression rank scores, score function, tail behavior 2008 MSC: 62E15, 62J05, 62G30, 62H10

\section{Introduction}

Consider the linear regression model

$$
Y_{i}=\mathbf{x}_{i}^{\top} \boldsymbol{\beta}+e_{i}, i=1, \ldots, n
$$

with an unknown parameter $\boldsymbol{\beta}$, where $\mathbf{x}_{i} \in \mathbb{R}^{p}, x_{i 1}=1, i=1, \ldots, n$ and where $e_{1}, \ldots, e_{n}$ are i.i.d. errors with distribution function $F$ and density $f$. We assume that the matrix $\mathbf{X}$ with the rows $\mathbf{x}_{1}^{\top}, \ldots, \mathbf{x}_{n}^{\top}$ is of rank $p$ and that $f(z)$ is absolutely continuous and positive for $z \in(a, b)$ where $a=\inf \{z: F(x)>0\}$ and $b=\sup \{z: F(z)<1\}$.

The regression $\alpha$-quantile $(0<\alpha<1)$ of Koenker and Bassett (1978) is any vector $\hat{\boldsymbol{\beta}}_{n}(\alpha)$ minimizing the criterion

$$
\sum_{i=1}^{n} \rho_{\alpha}\left(Y_{i}-\mathbf{x}_{i}^{\top} \mathbf{b}\right), \quad \mathbf{b} \in \mathbb{R}^{p}
$$

where $\rho_{\alpha}(z)=|z|\{\alpha I[z>0]+(1-\alpha) I[z<0]\}$. Dual to $\hat{\boldsymbol{\beta}}_{n}(\alpha)$ in the linear programming sense is the vector $\hat{\mathbf{a}}_{n}(\alpha)=\left(\hat{a}_{n 1}(\alpha), \ldots, \hat{a}_{n n} \alpha\right)^{\top}$ of the regression rank scores, defined as a solution of the linear programming problem

$$
\sum_{i=1}^{n} \hat{a}_{n i}(\alpha) Y_{i}=\max
$$

\footnotetext{
Research was supported by the Grants GAČR201/09/0133, IAA101120801, and by Research Projects MSM 0021620839 and LC 06024.

Preprint submitted to Statistics and Probability Letters

August 31, 2010
} 


$$
\begin{array}{ll}
\text { under } \quad & \sum_{i=1}^{n} \hat{a}_{n i}(\alpha)=n(1-\alpha) \\
& \sum_{i=1}^{n} x_{i j} \hat{a}_{n i}(\alpha)=(1-\alpha) \sum_{i=1}^{n} x_{i j}, \quad j=2, \ldots, p \\
& 0 \leq \hat{a}_{n i}(\alpha) \leq 1, \quad i=1, \ldots, n .
\end{array}
$$

Because of the restrictions in (3), the $\hat{a}_{n i}$ are unchanged if $Y_{i}$ is replaced with $e_{i}=Y_{i}-\mathbf{x}_{i}^{\top} \boldsymbol{\beta}, i=$ $1, \ldots, n$. As mutually dual, $\hat{\mathbf{a}}_{n}(\alpha)$ and $\hat{\boldsymbol{\beta}}_{n}(\alpha)$ are in the following relation:

$$
\hat{a}_{n i}(\alpha)=\left\{\begin{array}{ccc}
1 & \ldots & Y_{i}>\mathbf{x}_{i}^{\top} \hat{\boldsymbol{\beta}}(\alpha) \\
0 & \ldots & Y_{i}<\mathbf{x}_{i}^{\top} \hat{\boldsymbol{\beta}}(\alpha)
\end{array}, \quad i=1, \ldots, n,\right.
$$

while $0<\hat{a}_{n i}(\alpha)<1$ for $Y_{i}=\mathbf{x}_{i}^{\top} \hat{\boldsymbol{\beta}}_{n}(\alpha), i=1, \ldots, n$; the latter correspond to the optimal basis of $p$ vectors among $\mathbf{x}_{1}, \ldots, \mathbf{x}_{n}$. Moreover, the identity

$$
\sum_{i=1}^{n} \mathbf{x}_{i}^{\top} \hat{\boldsymbol{\beta}}(\alpha)=-\sum_{i=1}^{n} Y_{i} \frac{d \hat{a}_{i}(\alpha)}{d \alpha}
$$

holds at the point $\alpha$ of continuity of $\hat{\boldsymbol{\beta}}(\cdot)$ (see. e.g., Hallin and Jurečková (1999)). The derivative of $\hat{a}_{i}(\alpha)$ is nonzero only if $Y_{i}=\mathbf{x}_{i}^{\top} \hat{\boldsymbol{\beta}}(\alpha)$.

In the location model, where $\mathbf{X}=\mathbf{1}_{n}$, the regression $\alpha$-quantile reduces to the $\lceil n \alpha\rceil$-order statistic $Y_{n:[n \alpha]}$, and the vector of $\alpha$-regression rank scores reduces to the Hájek's rank scores which can be expressed as follows:

$$
a_{n i}^{*}\left(R_{i}, \alpha\right)= \begin{cases}0 & \ldots R_{i}<n \alpha \\ R_{i}-n \alpha & \ldots\left(R_{i}-1\right) / n<\alpha \leq R_{i} / n \\ 1 & \ldots \alpha \leq\left(R_{i}-1\right) / n \quad i=1, \ldots, n\end{cases}
$$

where $R_{i}$ is the rank of $Y_{i}$ among $Y_{1}, \ldots, Y_{n}$. Rank scores $a_{n}^{*}\left(R_{i}, \alpha\right)$ were first used by Hájek (1965) as a starting point for the construction of the nonlinear rank tests. We see that the identity (5) is also true in the location model, where $\frac{d \hat{a}_{i}(\alpha)}{d \alpha} \neq 0$ only for $i$ such that $n \alpha<R_{i}<n \alpha+1, i=1, \ldots, n$.

In the location model we have i.i.d. observations $Y_{1}, \ldots, Y_{n}$ with the ranks $R_{1}, \ldots, R_{n}$, and the vector of ranks is independent of the vector of order statistics $\mathbf{Y}_{(\cdot)}=\left(Y_{n: 1}, \ldots, Y_{n: n}\right), Y_{n: 1} \leq$ $\ldots \leq Y_{n: n}$. The $k$-th order statistic $Y_{n: k}$ has the density

$$
\frac{n !}{(k-1) !(n-k) !}(F(y))^{k-1}(1-F(y))^{n-k} f(y),
$$

while $P\left\{R_{1}=r_{1}, \ldots, R_{n}=r_{n}\right\}=\frac{1}{n !}$ for any permutation $r_{1}, \ldots, r_{n}$ of numbers $1,2, \ldots, n$.

Generally, if the vector $Y_{1}, \ldots, Y_{n}$ has density $p\left(y_{1}, \ldots, y_{n}\right)$, the ranks and order statistics can be mutually dependent; their joint distribution can be written in the following way:

$$
P\left(\mathbf{Y}_{(\cdot)} \in B, R_{1}=r_{1}, \ldots, R_{n}=r_{n}\right)=\int_{B} \ldots \int p\left(y_{n: r_{1}}, \ldots, y_{n: r_{n}}\right) d y_{n: 1} \ldots d y_{n: n} .
$$

for any Borel subset of the ordered quadrant of $\mathbb{R}^{n}$ and for any permutation $r_{1}, \ldots, r_{n}$. 
The asymptotic distribution of regression quantiles and of regression rank scores under $n \rightarrow \infty$ was studied by more authors, under various conditions on $f$ and on the regressors [let us mention Koenker and Bassett (1978), Ruppert and Carroll (1980), Gutenbrunner and Jurečková (1992), Gutenbrunner et al. (1993), among others]. The asymptotic properties of autoregression quantiles and the rank scores was studied by Koul and Saleh (1995). However, the proofs of the asymptotic distributions available in the literature impose various restrictions on the tails of the distribution of errors and on the regression matrix, even though the numerical evidence demonstrates that e.g. the regression rank scores tests work under quite general conditions. For instance, the asymptotic distribution of the regression rank scores criterion was proved only when either the tails of the basic distribution are lighter than those of the $t$-distribution with 4 degrees of freedom or when the score-generating function $\varphi:(0,1) \mapsto \mathbb{R}^{1}$ is zero outside $(\varepsilon, 1-\varepsilon) \subset(0,1)$ (cf. e.g. Gutenbrunner and Jurečková (1992), Gutenbrunner et al. (1993)). Moreover, the asymptotic distribution, which is typically normal, does not provide the full information on the behavior of $\hat{\boldsymbol{\beta}}(\alpha)$, and it can stretch its true behavior under heavy-tailed $f$.

We expect that the finite-sample distribution of the $\alpha$-regression quantile reminds the distribution of the sample quantile in the location model. Koenker and Bassett (1978) provided one possible form of the finite-sample distribution of $\hat{\boldsymbol{\beta}}(\alpha)$ (see also Koenker (2005)). However, these authors themselves do not find their form very tractable and adaptable to practical statistical inference. A possible form of the finite sample distribution, similar to the distribution of the sample quantile, is given in the present paper. Using the regression rank scores, dual to regression quantiles, we first derive the joint distribution of $\left(\hat{\mathbf{a}}_{n}(\alpha), \hat{\boldsymbol{\beta}}(\alpha)\right)$ in the linear regression model (1). Unlike in Koenker and Bassett (1978), our main tool is using the score function of $\left(\hat{\mathbf{a}}_{n}(\alpha), \hat{\boldsymbol{\beta}}(\alpha)\right)$ and the fact that the score function of a statistic $S=S(\mathbf{Y})$ is equal to the conditional expectation of the score function of $\mathbf{Y}$ under given $S$, provided it exists (see, e.g., Kagan et al. (1972) and Jurečková and Milhaud (2003)). We also benefit from the fact that the regression quantiles and rank scores are mutually dual optimal solutions of a linear programming problem. Because $0 \leq \hat{a}_{i}(\alpha) \leq 1, i=1, \ldots, n$, the vector $\mathbf{a}(\alpha)$ can be interpreted as a test function, and we can use the generalized Neyman-Pearson lemma.

\section{Distribution of $\alpha$-regression quantile under i.i.d. errors}

Consider the linear regression model (1) with deterministic regression matrix $\mathbf{X}$ with the first column equal to $\mathbf{1}_{n}$. Consider first the situation that the errors $e_{1}, \ldots, e_{n}$ are i.i.d. with distribution function $F$ and density $f$. We assume that the matrix $\mathbf{X}$ with the rows $\mathbf{x}_{1}^{\top}, \ldots, \mathbf{x}_{n}^{\top}$ has the rank $p$ and that $f(z)$ is absolutely continuous and positive for $z \in(a, b)$ where $a=\inf \{z: F(x)>0\}$ and $b=\sup \{z: F(z)<1\}$. The first step is to derive the score function of $(\hat{\mathbf{a}}(\alpha), \hat{\boldsymbol{\beta}}(\alpha))$.

Generally, the score function of the vector $Y_{1}, \ldots, Y_{n}$ with density $p\left(y_{1}, \ldots, y_{n}, \boldsymbol{\theta}\right)=p(\mathbf{y}, \boldsymbol{\theta})$, $\boldsymbol{\theta} \in \boldsymbol{\Theta} \subset \mathbb{R}^{p}$, is the vector function

$$
\left(\frac{\partial \ln p(\mathbf{y}, \boldsymbol{\theta})}{\partial \theta_{j}}, j=1, \ldots, p\right)^{\top} .
$$

The score function is in the 1:1 correspondence with the probability distribution, and it is one of its main characteristics. Using the score functions was recommended by Hampel (1973), Field and Hampel (1982), Field and Ronchetti (1990), among others. A natural argument for the use of the score function is that it is linear for the normal distribution, which is considered as a unit in the family of probability distributions. 
Fix $\alpha$ and consider the set

$$
\begin{aligned}
\mathbf{A}_{n}(\alpha)= & \left\{\mathbf{a}: 0 \leq a_{i} \leq 1, i=1, \ldots, n, \sum_{i=1}^{n} \mathbf{x}_{i} a_{i} I\left[a_{i}>0\right]=(1-\alpha) \sum_{i=1}^{n} \mathbf{x}_{i}\right. \\
& \text { and } 0<a_{i_{j}}<1, j=1, \ldots, p \text { for } 1 \leq i_{1}, \ldots, i_{p} \leq n, \\
& \text { such that } \left.\quad \mathbf{x}_{i_{1}}, \ldots, \mathbf{x}_{i_{p}} \text { is a basis of } \mathbb{R}^{p}\right\} .
\end{aligned}
$$

The score function of the vector $(\hat{\mathbf{a}}(\alpha), \hat{\boldsymbol{\beta}}(\alpha))$ follows.

Lemma 2.1. Under the conditions stated above, the score function of $(\hat{\mathbf{a}}(\alpha), \hat{\boldsymbol{\beta}}(\alpha))$ has the form

$$
\begin{gathered}
\mathbf{r}(\mathbf{a}, \mathbf{b}, \boldsymbol{\beta})=-\sum_{i=1}^{n} \mathbf{x}_{i}\left\{f\left(\mathbf{x}_{i}^{\top}[\mathbf{b}-\boldsymbol{\beta}]\right)\left(\frac{I\left[a_{i}=0\right]}{F\left(\mathbf{x}_{i}^{\top}[\mathbf{b}-\boldsymbol{\beta}]\right)}-\frac{I\left[a_{i}=1\right]}{1-F\left(\mathbf{x}_{i}^{\top}[\mathbf{b}-\boldsymbol{\beta}]\right)}\right)\right. \\
\left.+I\left[0<a_{i}<1\right] \frac{f^{\prime}\left(\mathbf{x}_{i}^{\top}[\mathbf{b}-\boldsymbol{\beta}]\right)}{f\left(\mathbf{x}_{i}^{\top}[\mathbf{b}-\boldsymbol{\beta}]\right.}\right\}, \quad \mathbf{a} \in \mathbf{A}_{n}(\alpha), \mathbf{b} \in \mathbb{R}^{p} .
\end{gathered}
$$

Proof. The score function of model (1) is the vector function

$$
\begin{aligned}
& \left(\frac{\partial \ln \prod_{i=1}^{p} f\left(y_{i}-\mathbf{x}_{i}^{\top} \beta\right)}{\partial \beta_{j}}, j=1, \ldots, p\right)^{\top} \\
& =\left(\frac{\sum_{i=1}^{n} \partial \ln f\left(y_{i}-\mathbf{x}_{i}^{\top} \boldsymbol{\beta}\right)}{\partial \beta_{j}}, j=1, \ldots, p\right)^{\top}=-\sum_{i=1}^{n} \mathbf{x}_{i} \frac{f^{\prime}\left(y_{i}-\mathbf{x}_{i}^{\top} \beta\right)}{f\left(y_{i}-\mathbf{x}_{i}^{\top} \beta\right)} .
\end{aligned}
$$

Regarding the inequalities (4), the conditional distribution of $Y_{i}$, given $\left[\hat{a}_{i}(\alpha)=0 \Leftrightarrow Y_{i}<\right.$ $\left.\mathbf{x}_{i}^{\top} \hat{\boldsymbol{\beta}}(\alpha)\right]$ and $[\hat{\boldsymbol{\beta}}(\alpha)=\mathbf{b}]$ has the density

$$
\frac{f\left(y-\mathbf{x}_{i}^{\top} \boldsymbol{\beta}\right)}{\left.F\left(\mathbf{x}_{i}^{\top}[\mathbf{b}-\boldsymbol{\beta}]\right)\right)} I\left[\hat{a}_{n i}(\alpha)=0\right] .
$$

Similarly, the conditional density of $Y_{i}$ given $\left[\hat{a}_{i}(\alpha)=0 \Leftrightarrow Y_{i}>\mathbf{x}_{i}^{\top} \hat{\boldsymbol{\beta}}(\alpha)\right]$ and $[\hat{\boldsymbol{\beta}}(\alpha)=\mathbf{b}]$ is

$$
\frac{f\left(y-\mathbf{x}_{i}^{\top} \boldsymbol{\beta}\right)}{\left.1-F\left(\mathbf{x}_{i}^{\top}[\mathbf{b}-\boldsymbol{\beta}]\right)\right)} I\left[\hat{a}_{n i}(\alpha)=1\right] .
$$

This further implies for $i=1, \ldots, n$

$$
\begin{aligned}
& \mathbb{E}_{\beta}\left\{\left(-\frac{f^{\prime}\left(Y_{i}-\mathbf{x}_{i}^{\top} \boldsymbol{\beta}\right)}{f\left(Y_{i}-\mathbf{x}_{i}^{\top} \boldsymbol{\beta}\right)}\right) \mid \hat{\boldsymbol{\beta}}_{n}(\alpha)=\mathbf{b}, \hat{a}_{n i}(\alpha)=0\right\} \\
& =\frac{1}{F\left(\mathbf{x}_{i}^{\top}[\mathbf{b}-\boldsymbol{\beta}]\right)} \int_{-\infty}^{\mathbf{x}_{i}^{\top} \mathbf{b}}\left(-\frac{f^{\prime}\left(y-\mathbf{x}_{i}^{\top} \boldsymbol{\beta}\right)}{f\left(y-\mathbf{x}_{i}^{\top} \boldsymbol{\beta}\right)}\right) f\left(y-\mathbf{x}_{i}^{\top} \boldsymbol{\beta}\right) d y=\frac{-f\left(\mathbf{x}_{i}^{\top}[\mathbf{b}-\boldsymbol{\beta}]\right)}{F\left(\mathbf{x}_{i}^{\top}[\mathbf{b}-\boldsymbol{\beta}]\right)}
\end{aligned}
$$


and similarly,

$$
\begin{aligned}
& \mathbb{E}_{\beta}\left\{\left(-\frac{f^{\prime}\left(Y_{i}-\mathbf{x}_{i}^{\top} \boldsymbol{\beta}\right)}{f\left(Y_{i}-\mathbf{x}_{i}^{\top} \boldsymbol{\beta}\right)}\right) \mid \hat{\boldsymbol{\beta}}_{n}(\alpha)=\mathbf{b}, \hat{a}_{n i}(\alpha)=1\right\} \\
& =\frac{1}{1-F\left(\mathbf{x}_{i}^{\top}[\mathbf{b}-\boldsymbol{\beta}]\right)} \int_{\mathbf{x}_{i}^{\top} \mathbf{b}}^{\infty}\left(-\frac{f^{\prime}\left(y-\mathbf{x}_{i}^{\top} \boldsymbol{\beta}\right)}{f\left(y-\mathbf{x}_{i}^{\top} \boldsymbol{\beta}\right)}\right) f\left(y-\mathbf{x}_{i}^{\top} \boldsymbol{\beta}\right) d y=\frac{f\left(\mathbf{x}_{i}^{\top}[\mathbf{b}-\boldsymbol{\beta}]\right)}{1-F\left(\mathbf{x}_{i}^{\top}[\mathbf{b}-\boldsymbol{\beta}]\right)} .
\end{aligned}
$$

Moreover,

$$
\mathbb{E}_{\beta}\left\{\left(-\frac{f^{\prime}\left(Y_{i}-\mathbf{x}_{i}^{\top} \boldsymbol{\beta}\right)}{f\left(Y_{i}-\mathbf{x}_{i}^{\top} \boldsymbol{\beta}\right)}\right) \mid \hat{\boldsymbol{\beta}}_{n}(\alpha)=\mathbf{b}, 0<\hat{a}_{n i}(\alpha)<1\right\}=-\frac{f^{\prime}\left(\mathbf{x}_{i}^{\top}[\mathbf{b}-\boldsymbol{\beta}]\right)}{f\left(\mathbf{x}_{i}^{\top}[\mathbf{b}-\boldsymbol{\beta}]\right)},
$$

The score function of $\left(\hat{\mathbf{a}}_{n}(\alpha), \hat{\boldsymbol{\beta}}_{n}(\alpha)\right)$ follows from (10), (11), (12).

Integrating (8) over $\beta$, we obtain the joint distribution of the $\alpha$-regression rank scores $\hat{\mathbf{a}}_{n}(\alpha)$ and of the $\alpha$-regression quantile $\hat{\boldsymbol{\beta}}_{n}(\alpha)$ :

Lemma 2.2. Under the above conditions, the joint distribution of the $\alpha$-regression rank score and $\alpha$-regression quantile has the form

$$
\begin{aligned}
& P_{\beta}\left\{\hat{\boldsymbol{\beta}}_{n}(\alpha) \in \mathbf{B}, \hat{\mathbf{a}}_{n}(\alpha)=\mathbf{a}\right\} \\
& =C \int_{\mathbf{B}} \prod_{i=1}^{n}\left[\left(F\left(\mathbf{x}_{i}^{\top}[\mathbf{b}-\boldsymbol{\beta}]\right)\right)^{I\left[a_{i}=0\right]}\left(1-F\left(\mathbf{x}_{i}^{\top}[\mathbf{b}-\boldsymbol{\beta}]\right)^{I\left[a_{i}=1\right]}\left(f\left(\mathbf{x}_{i}^{\top}[\mathbf{b}-\boldsymbol{\beta}]\right)\right)^{I\left[0<a_{i}<1\right]}\right] d \mathbf{b},\right.
\end{aligned}
$$

$\mathbf{a} \in \mathbf{A}_{n}(\alpha), \mathbf{B} \in \mathcal{B}^{p}, C>0$ is a normalizing constant.

Proof. Denote $\mathcal{H}=\left\{\left(\sum_{i=1}^{n} x_{i 1} a_{i}, \sum_{i=1}^{n} x_{i 2} a_{i}, \ldots, \sum_{i=1}^{n} x_{i p} a_{i}\right)^{\top}: 0 \leq a_{i} \leq 1, i=1, \ldots, n\right\}$. Then $\mathcal{H}$ is closed and convex. Let $\mathbf{a} \in \mathbf{A}_{n}(\alpha), 0<a_{i_{j}}<1$ for $j=1, \ldots, p$, and let $\mathbf{x}_{i_{1}}^{\top}, \ldots, \mathbf{x}_{i_{p}}^{\top}$ be linearly independent rows of matrix $\mathbf{X}$. Fix $\mathbf{b} \in \mathbb{R}^{p}, \mathbf{b} \neq \mathbf{0}$, and choose $y_{1}, \ldots, y_{n}$ so that

$$
\begin{aligned}
& y_{i_{j}}=\mathbf{x}_{i_{j}}^{\top} \mathbf{b}, j=1, \ldots, p \\
& y_{i}>\mathbf{x}_{i}^{\top} \mathbf{b} \text { if } a_{i}=1, \\
& y_{i}<\mathbf{x}_{i}^{\top} \mathbf{b} \text { if } a_{i}=0, \quad i=1, \ldots, n .
\end{aligned}
$$

Then a maximizes $\sum_{i=1}^{n} y_{i} a_{i}$ under $\mathbf{a} \in \mathbf{A}_{n}(\alpha)$, and $\mathbf{b}$ is the $\alpha$-regression quantile pertaining to $\left(y_{1}, \ldots, y_{n}\right)$. On the other hand, let a solve (3). Because $(1-\alpha) \sum_{i=1}^{n} \mathbf{x}_{i}$ is an inner point of $\mathcal{H}$, there exists the vector $\mathbf{b}$ of Lagrange multipliers such that

$$
\begin{aligned}
& y_{i}>\mathbf{x}_{i}^{\top} \mathbf{b} \text { if } a_{i}=1, \\
& y_{i}<\mathbf{x}_{i}^{\top} \mathbf{b} \text { if } a_{i}=0, \quad i=1, \ldots, n,
\end{aligned}
$$

the remaining $a_{i}$ corresponding to $y_{i}=\mathbf{x}_{i}^{\top} \mathbf{b}$ and the values of $\mathbf{b}$ are determined so that $\sum_{i=1}^{n} \mathbf{x}_{i} a_{i}=$ $(1-\alpha) \sum_{i=1}^{n} \mathbf{x}_{i}$ (cf. the Neyman-Pearson lemma [Lemma 3 in (18), Chapter 3]).

Lemmas 2.1 and 2.2 finally give the density of the $\alpha$-regression quantile. 
Theorem 2.3. Consider the linear regression model (1) with deterministic regression matrix $\mathbf{X}$ of the rank $p$, with the first column equal to $\mathbf{1}_{n}$. Assume that the errors $e_{1}, \ldots, e_{n}$ are i.i.d. with absolutely continuous distribution function $F$ and with density $f$, absolutely continuous and positive for $z \in(a, b)$ where $a=\inf \{z: F(x)>0\}$ and $b=\sup \{z: F(z)<1\}$. Then the $\alpha$-regression quantile $\hat{\boldsymbol{\beta}}(\alpha), 0<\alpha<1$ has the density

$$
\begin{aligned}
g(\mathbf{b} ; \alpha) & =\sum_{\mathbf{a} \in \mathbf{A}_{n}(\alpha)} \prod_{i=1}^{n}\left[\left(F\left(\mathbf{x}_{i}^{\top}[\mathbf{b}-\boldsymbol{\beta}]\right)\right)^{I\left[a_{i}=0\right]}\left(1-F\left(\mathbf{x}_{i}^{\top}[\mathbf{b}-\boldsymbol{\beta}]\right)^{I\left[a_{i}=1\right]}\left(f\left(\mathbf{x}_{i}^{\top}[\mathbf{b}-\boldsymbol{\beta}]\right)\right)^{I\left[0<a_{i}<1\right]}\right],\right. \\
& \mathbf{b} \in \mathbb{R}^{p} .
\end{aligned}
$$

\section{Remarks and application to the tail behavior of regression quantiles}

In the location model with $\mathbf{X}=\mathbf{1}_{n}$, density (14) reduces to the density of the order statistic $Y_{n: k}$ with $k=\lceil n \alpha\rceil$. Indeed, then $\mathbf{X}_{n} \equiv \mathbf{1}_{n}$ and the regression rank scores reduce to the Hájek scores $(6)$. Then $\hat{a}_{n i}(\alpha) \in(0,1)$ iff the rank of $Y_{i}$ equals $R_{i}=\lceil n \alpha\rceil$, what happens just for one among $Y_{1}, \ldots, Y_{n}$ with probability 1 , and $\hat{a}_{n k}(\alpha)=0$ or 1 according to whether $R_{k}$ is greater or less than $\lceil n \alpha\rceil$, respectively.

Even in the location model the moments of order statistics must be calculated numerically. In spite of that, the BLUE estimators of Lloyd (1952) [see also Sarhan and Greenberg (1962), David (1981)] are considered useful in various contexts. For instance, the Shapiro-Wilk (1965) test is a surprisingly good intuitive test of normality based on the ratio of the MLE and BLUE estimators of $\sigma$ under normal $f$. Sen et al. (2003) extended the test to the model with the nuisance linear regresssion, where it also works well.

With the above methods we can derive the joint distribution of two regression quantiles, $\hat{\boldsymbol{\beta}}\left(\alpha_{1}\right), \hat{\boldsymbol{\beta}}\left(\alpha_{2}\right)$. Their expectations and covariances are sufficient just under $\boldsymbol{\beta}=\mathbf{0}$, because of the affine equivariance of regression quantiles and of the invariance of regression rank scores.

Even if the finite sample distribution is more complex than the asymptotic one, and the finite sample moments are calculated via the numerical integration, we cannot limit ourselves to the asymptotics, which can stretch the truth and is sometimes informative only for a very large number of observations.

As an illustration, we apply the methods of Section 2 to the study of the finite sample tail behavior of regression quantiles. Typically, the quantiles and other estimators inherit their tail behavior from the parent distribution, though their asymptotic (normal) distribution is light-tailed. Following He et al. (1990), consider the following finite-sample measure of the right-tail performance of a regression estimator $\mathbf{T}_{n}$ in model (1) (assume $0<F<1, f>0$, for the brevity)

$$
B\left(\mathbf{T}_{n}, \gamma\right)=\frac{-\ln P_{\beta}\left(\max _{1 \leq i \leq n}\left\{\mathbf{x}_{i}^{\top}\left(\mathbf{T}_{n}-\boldsymbol{\beta}\right)\right\}>\gamma\right)}{-\ln (1-F(\gamma))}, \quad \gamma>0 .
$$

The probability in (15) tends to 0 as $\gamma \rightarrow \infty$, but the rate of this convergence depends on the tail of $F$. Then the bounds of (15) under finite $n$ and $\gamma \rightarrow \infty$ are of interest, because they show how faster is the convergence $P_{\beta}\left(\max _{1 \leq i \leq n}\left\{\mathbf{x}_{i}^{\top}\left(\mathbf{T}_{n}-\boldsymbol{\beta}\right)\right\}>\gamma\right) \rightarrow 0$ than $1-F(\gamma) \rightarrow 0$ as $\gamma \rightarrow \infty$.

Put $\boldsymbol{\beta}=\mathbf{0}$, without loss of generality, and assume for simplicity that $n \alpha=k>0$, an integer, and $k+p<n$. Regarding (3) and (4), we can write

$$
P_{0}\left(\max _{i}\left\{\mathbf{x}_{i}^{\top} \hat{\boldsymbol{\beta}}(\alpha)\right\} \leq \gamma\right) \leq P_{0}(\text { at least } k+1 \text { among } Y \text { 's are } \leq \gamma)
$$


hence

$$
\begin{aligned}
& P_{0}\left(\max _{i} \mathbf{x}_{i}^{\top} \hat{\boldsymbol{\beta}}(\alpha)>\gamma\right) \geq P_{0}\left(Y_{n: k+1}>\gamma\right)=\frac{n !}{k !(n-k-1) !} \int_{F(\gamma)}^{1} u^{k}(1-u)^{n-k-1} d u \\
& \geq \frac{n !}{k !(n-k-1) !}(F(\gamma))^{k} \int_{0}^{1-F(\gamma)} v^{n-k-1} d v=\frac{n !}{k !(n-k) !}(F(\gamma))^{k}(1-F(\gamma))^{n-k} .
\end{aligned}
$$

It implies

$$
\varlimsup_{\gamma \rightarrow \infty} \frac{-\ln P_{0}\left(\max _{i} \mathbf{x}_{i}^{\top} \hat{\boldsymbol{\beta}}(\alpha)>\gamma\right)}{-\ln (1-F(\gamma))} \leq n-k
$$

Similarly,

$$
P_{0}\left(\max _{i} \mathbf{x}_{i}^{\top} \hat{\boldsymbol{\beta}}(\alpha) \leq \gamma\right) \geq P_{0}\left(Y_{n: k+p} \leq \gamma\right)
$$

hence

$$
\begin{aligned}
& P_{0}\left(\max _{i} \mathbf{x}_{i}^{\top} \hat{\boldsymbol{\beta}}(\alpha)>\gamma\right) \leq \frac{n !}{(k+p-1) !(n-k-p) !} \int_{F(\gamma)}^{1} u^{k+p-1}(1-u)^{n-k-p} d u \\
& \frac{n !}{(k+p-1) !(n-k-p) !}(1-F(\gamma))^{n-k-p}\left(1-(F(\gamma))^{k+p}\right) \leq \frac{n !(k+p-1)}{(k+p) !(n-k-p) !}(1-F(\gamma))^{n-k-p+1}
\end{aligned}
$$

and this implies

$$
\lim _{\gamma \rightarrow \infty} \frac{-\ln P_{0}\left(\left(\max _{i} \mathbf{x}_{i}^{\top} \hat{\boldsymbol{\beta}}(\alpha)>\gamma\right)\right.}{-\ln (1-F(\gamma))} \geq n-k-p+1
$$

Summarizing, we conclude

$$
n-k-p+1 \leq \underline{\lim }_{\gamma \rightarrow \infty} B(\hat{\boldsymbol{\beta}}(\alpha), \gamma) \leq \varlimsup_{\gamma \rightarrow \infty} B(\hat{\boldsymbol{\beta}}(\alpha), \gamma) \leq n-k
$$

what is consistent with the tail behavior of the order statistic $Y_{n: k}$ in the location model (where $p=1)$.

\section{Distribution of the extreme regression quantile}

After a slight modification, the above method applies to the extreme regression quantiles, corresponding to $\alpha=0,1$. The maximal regression quantile $\hat{\boldsymbol{\beta}}(1)$ is a solution of the minimization problem:

$$
\min _{\mathbf{b} \in \mathbb{R}^{p}} \sum_{i=1}^{n}\left(Y_{i}-\mathbf{x}_{i}^{\top} \mathbf{b}\right)^{+}
$$

where $z^{+}=\max (z, 0)$ denotes the positive part of $z$. The minimization (17) can be alternatively described as any solution to the linear program:

$$
\min _{\mathbf{b} \in \mathbb{R}^{p}} \sum_{i=1}^{n}\left\{\mathbf{x}_{i}^{\top} \mathbf{b}\right\} \quad \text { s.t. } \quad Y_{i} \leq \mathbf{x}_{i}^{\top} \mathbf{b}, \quad i=1, \ldots, n .
$$

This statistic was studied by Smith (1994) who derived its asymptotic distribution under heavytailed distribution $F$ and under some conditions on the $\mathbf{x}_{i}$. Portnoy and Jurečková (1999), Knight 
(2001), Chernozhukov (2005), Jurečková (2007) (among others), derived various forms of the asymptotic distributions of the extreme regression quantiles under various conditions. As in the extreme value theory, the asymptotic distribution of the extreme regression quantile depends on the domain of attraction of the model errors, and the results in the literature are proved under various additional conditions on $f$ and on $\mathbf{X}$.

If $\left(\mathbf{x}_{i_{1}}, \ldots, \mathbf{x}_{i_{p}}\right)^{\top}=\tilde{\mathbf{x}}$ is the optimal basis of the problem (18), then $Y_{i_{j}}=\mathbf{x}_{i_{j}}^{\top} \hat{\boldsymbol{\beta}}(1)$ for $j=$ $1, \ldots, p$ and $Y_{i}<\mathbf{x}_{i}^{\top} \hat{\boldsymbol{\beta}}(1)$ for the remaining components. Denote the set

$$
\begin{aligned}
C_{n}= & \left\{\mathbf{c}=\left(c_{1}, \ldots, c_{n}\right)^{\top}: c_{i_{1}}, \ldots, c_{i_{p}}=1 \text { for } 1 \leq i_{1}<\ldots<i_{p} \leq n\right. \\
& \text { such that } \left.\left(\mathbf{x}_{i_{1}}, \ldots, \mathbf{x}_{i_{p}}\right) \text { is a basis of } \mathbb{R}^{p} \text {, and } c_{i}=0 \text { otherwise. }\right\}
\end{aligned}
$$

Proceeding analogously as in Lemmas 2.1, 2.2 and Theorem 2.3, we obtain the density of the extreme regression quantile:

Theorem 4.1. Under the conditions of Theorem 2.3, the extreme regression quantile $\hat{\boldsymbol{\beta}}(1)$ has the density

$$
g(\mathbf{b} ; 1)=\sum_{\mathbf{c} \in \mathbf{C}_{n}} \prod_{i=1}^{n}\left[\left(F\left(\mathbf{x}_{i}^{\top}[\mathbf{b}-\boldsymbol{\beta}]\right)\right)^{I\left[c_{i}=0\right]}\left(f\left(\mathbf{x}_{i}^{\top}[\mathbf{b}-\boldsymbol{\beta}]\right)\right)^{I\left[c_{i}=1\right]}\right], \quad \mathbf{b} \in \mathbb{R}^{p} .
$$

Proof. The optimal solution $\hat{\boldsymbol{\beta}}(1)=\mathbf{b}$ of (18) determines the optimal basis $\left(\mathbf{x}_{i_{1}}, \ldots, \mathbf{x}_{i_{p}}\right)$ and $Y_{i_{j}}=\mathbf{x}_{i_{j}}^{\top} \mathbf{b}, j=1, \ldots, p$. For the remaining $i$ we have the conditional probability

$$
P\left(Y_{i}<y \mid \hat{\boldsymbol{\beta}}(1)=\mathbf{b}\right)=\frac{\left.F\left(y-\mathbf{x}_{i}^{\top} \boldsymbol{\beta}\right)\right)}{F\left(x_{i}^{\top}(\mathbf{b}-\boldsymbol{\beta})\right.} I\left[Y_{i}<\mathbf{x}_{i}^{\top} \mathbf{b}\right] .
$$

Following the steps in the proofs of Lemmas 2.1, 2.2 and Theorem 2.3, we arrive at (20). In the location model with $\mathbf{X}=\mathbf{1}_{n}$, density (20) reduces to $n(F(x))^{n-1} f(x)$, the density of the maximal order statistic.

\section{Distribution of $\alpha$-regression quantile under non-i.i.d. errors}

The distribution of the regression quantile can be obtained even when the errors $e_{i}$ are nonidentically distributed, but still independent. Consider the model (1) with the change that the error $e_{i}$ has a distribution function $F_{i}$ with density $f_{i}$, hence $Y_{i}$ has distribution function $F_{i}\left(y-\mathbf{x}_{i}^{\top} \boldsymbol{\beta}\right), i=$ $1, \ldots, n$. The distribution functions $F_{1}, \ldots, F_{n}$ are generally different, but all are assumed to be absolutely continuous and strictly increasing on $\mathbb{R}^{1}$ with absolutely continuous densities. A special case is the heteroscedastic model

$$
Y_{i}=\mathbf{x}_{i}^{\top} \boldsymbol{\beta}+\sigma_{i} e_{i}
$$

with $e_{1}, \ldots, e_{n}$ i.i.d. with distribution function $F$, i.e. $F_{i}(z)=F\left(\frac{z}{\sigma_{i}}\right), i=1, \ldots, n$.

The score function of the random vector $\left(Y_{1}, \ldots, Y_{n}\right)$ in the non-i.i.d. model is

$$
-\sum_{i=1}^{n} \mathbf{x}_{i} \frac{f_{i}^{\prime}\left(y_{i}-\mathbf{x}_{i}^{\top} \boldsymbol{\beta}\right)}{f_{i}\left(y_{i}-\mathbf{x}_{i}^{\top} \boldsymbol{\beta}\right)}
$$


The definitions (2) and (3) of the regression quantile and of the regression rank scores are unchanged, and so are their algebraic properties. So, following the steps of proofs of Lemmas 2.1 and 2.2, we shall arrive in the following form of distribution of the regression quantile in this situation:

Theorem 5.1. Consider the linear regression model (1) with the deterministic regression matrix $\mathbf{X}$ of the rank $p$, with the first column equal to $\mathbf{1}_{n}$. Assume that the errors $e_{1}, \ldots, e_{n}$ are independent with absolutely continuous and strictly increasing distribution functions $F_{i}, 0<F_{i}(x)<$ $1, x \in \mathbb{R}$, which have absolutely continuous densities $f_{i}>0, i=1, \ldots, n$. Then the $\alpha$-regression quantile $\hat{\boldsymbol{\beta}}^{*}(\alpha), 0<\alpha<1$ has the density

$$
\begin{aligned}
g^{*}(\mathbf{b} ; \alpha) & =\sum_{\mathbf{a} \in \mathbf{A}_{n}(\alpha)} \prod_{i=1}^{n}\left[\left(F_{i}\left(\mathbf{x}_{i}^{\top}[\mathbf{b}-\boldsymbol{\beta}]\right)\right)^{I\left[a_{i}=0\right]}\left(1-F_{i}\left(\mathbf{x}_{i}^{\top}[\mathbf{b}-\boldsymbol{\beta}]\right)^{I\left[a_{i}=1\right]}\left(f_{i}\left(\mathbf{x}_{i}^{\top}[\mathbf{b}-\boldsymbol{\beta}]\right)\right)^{I\left[0<a_{i}<1\right]}\right],\right. \\
& \mathbf{b} \in \mathbb{R}^{p} .
\end{aligned}
$$

The distribution of $\alpha$-regression quantile in the heteroscedastic model we obtain as a corollary.

\section{Concluding remarks}

The finite sample distributions of the $\alpha$-regression quantile and of the extreme regression quantile are analogous to the corresponding distributions in the location model; this again confirms that the regression quantile is an extension of the sample quantile. Moreover, these distributions are true for a broad class of underlying distributions of errors, even in the non-i.i.d. case, while the asymptotic distributions of regression quantiles were derived only under various restrictive conditions.

\section{References}

[1] Chernozhukov, V. (2005). Extremal quantile regression. Ann. Statist. 33, 806-839.

[2] David, H. A. (1981). Order Statistics, 2nd ed. J. Wiley, New York.

[3] Field, C. A. and Hampel, F. R. (1982). Small-sample asymptotic distribution of M-estimators of location. Biometrika 69, 29-46.

[4] Field, C. A. and Ronchetti, E. (1990). Small Sample Asymptotics. IMS Lecture Notes, Monograph Series, 13 Hayward, CA.

[5] Gutenbrunner, C. and Jurečková, J. (1992). Regression rank scores and regression quantiles. Ann. Statist. 20 305330.

[6] Gutenbrunner, C., Jurečková, J. Koenker, R. and Portnoy, S. (1993). Tests of linear hypotheses based on regression rank scores. Nonpar. Statist. 2 307-331.

[7] Hájek, J. (1965). Extension of the Kolmogorov-Smirnov test to the regression alternatives. Bernoulli-BayesLaplace, Proc. Intern. Res. Seminar (J.Neyman and L.LeCam, eds.), pp.45-60. Springer-Verlag, Berlin.

[8] Hallin, M. and Jurečková, J. (1999). Optimal tests for autoregressive models based on autoregression rank scores. Ann. Statist. 27, 1385-1414.

[9] Hampel, F.R. Some small-sample asymptotics. In: Prof. Prague Symposium on Asymptotic Statistics (J. Hájek, ed.), pp. 109-126. Charles University in Prague.

[10] He, X., Jurečková, J., Koenker, R. and Portnoy, S. (1990). ail behavior of regression estimators and their breakdown points. Econometrica 58, 1195-1214.

[11] Jurečková, J. (2007). Remark on extreme regression quantile. Sankhya 69, Part 1, 87-100.

[12] Jurečková, J. and Milhaud, X. (2003). Derivative in the mean of a density and statistical applications. In: IMS Lecture Notes, Monograph Series, 42, (M.Moore, S. Froda, C. Léger, eds.), pp. 216-230. Hayward, CA. 
[13] Kagan, A. M., Linnik, Ju. V. and Rao, C.R. (1972). Characteristic Problems of Mathematical Statistics. Nauka, Moscow.

[14] Knight, K. (2001). Limiting distributions for linear programming estimators. Extremes 4, 87-103.

[15] Koenker, R. (2005). Quantile Regression. Cambridge University Press, Cambridge.

[16] Koenker, R. and Bassett, G. (1978). Regression quantiles. Econometrica 46 466-476.

[17] Koul, H. L. and A.K.Md.E. Saleh (1995). Autoregression quantiles and related rank scores processes. Ann. Statist. 23, 670-689.

[18] Lehmann, E.L. (1986). Testing Statistical Hypotheses (Second Edition). Springer-Verlag, New York.

[19] Lloyd, E.H. (1952). Least squares estimation of location and scale parameters using order statistics. Biomatrika 34, 41-67.

[20] Portnoy, S. and J. Jurečková (1999). On extreme regression quantiles. Extremes 2, 227-243.

[21] Ruppert, D. and Carroll, R. J. (1980). Trimmed least squares estimation in the linear model. J. Amer. Statist. Assoc. 75, 828-838.

[22] Sarhan, A. E. and Greenberg, B. G. (eds.) (1962). Contributions to Order Statistics. J. Wiley, New York.

[23] Sen, P. K., Jurečková, J. and Picek, J. (2003). Goodness-of-fit test of Shapiro-Wilk type with nuisance regression and scale. Austrian J. of Statist. 32, No 1\& 2, 163-177.

[24] Shapiro, S. S. and Wilk, M.B. (1965). An analysis of variance for normality (complete samples). Biometrika 52 , 591-611.

[25] Smith, R. (1994). Nonregular regression. Biometrika 81, 173-183. 